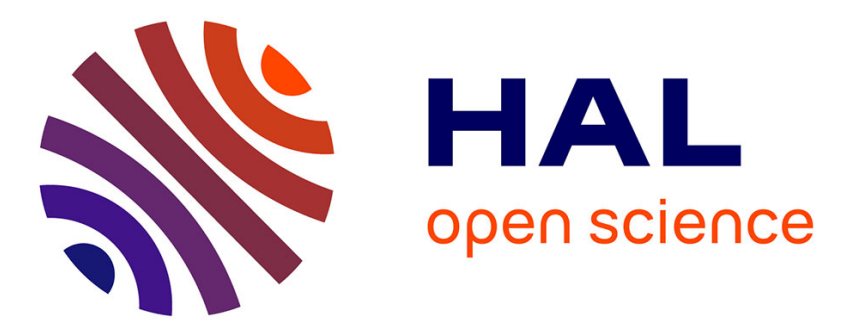

\title{
Hybrid stochastic-deterministic calculation of the second-order perturbative contribution of multireference perturbation theory
}

Yann Garniron, Anthony Scemama, Pierre-François Loos, Michel Caffarel

\section{- To cite this version:}

Yann Garniron, Anthony Scemama, Pierre-François Loos, Michel Caffarel. Hybrid stochasticdeterministic calculation of the second-order perturbative contribution of multireference perturbation theory. Journal of Chemical Physics, 2017, 147 (3), pp.034101. 10.1063/1.4992127 . hal-01539064

\section{HAL Id: hal-01539064 \\ https://hal.science/hal-01539064}

Submitted on 28 Jan 2020

HAL is a multi-disciplinary open access archive for the deposit and dissemination of scientific research documents, whether they are published or not. The documents may come from teaching and research institutions in France or abroad, or from public or private research centers.
L'archive ouverte pluridisciplinaire HAL, est destinée au dépôt et à la diffusion de documents scientifiques de niveau recherche, publiés ou non, émanant des établissements d'enseignement et de recherche français ou étrangers, des laboratoires publics ou privés. 


\section{Hybrid stochastic-deterministic calculation of the second-order perturbative contribution of multireference perturbation theory}

Cite as: J. Chem. Phys. 147, 034101 (2017); https://doi.org/10.1063/1.4992127

Submitted: 14 March 2017 . Accepted: 26 June 2017 . Published Online: 17 July 2017

Yann Garniron (D), Anthony Scemama (D), Pierre-François Loos, and Michel Caffarel (D)

\section{ARTICLES YOU MAY BE INTERESTED IN}

Selected configuration interaction method using sampled first-order corrections to wave functions

The Journal of Chemical Physics 147, 034102 (2017); https://doi.org/10.1063/1.4993214

Iterative perturbation calculations of ground and excited state energies from multiconfigurational zeroth-order wavefunctions

The Journal of Chemical Physics 58, 5745 (1973); https://doi.org/10.1063/1.1679199

Incremental full configuration interaction

The Journal of Chemical Physics 146, 104102 (2017); https://doi.org/10.1063/1.4977727

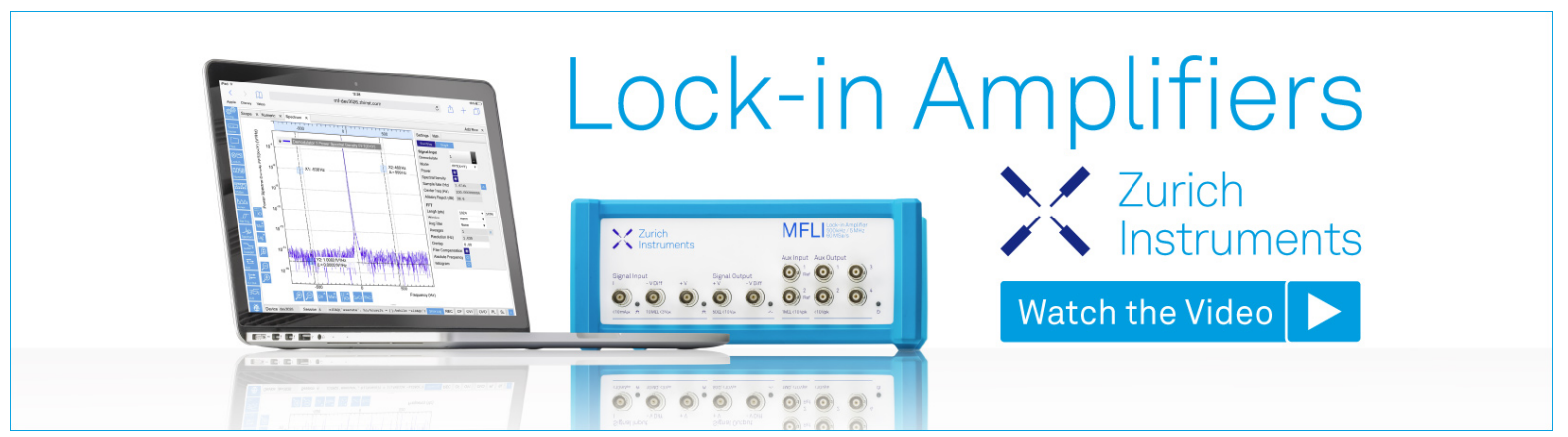




\title{
Hybrid stochastic-deterministic calculation of the second-order perturbative contribution of multireference perturbation theory
}

\author{
Yann Garniron, Anthony Scemama, ${ }^{\text {a) }}$ Pierre-François Loos, and Michel Caffarel \\ Laboratoire de Chimie et Physique Quantiques, Université de Toulouse, CNRS, UPS, Toulouse, France
}

(Received 14 March 2017; accepted 26 June 2017; published online 17 July 2017)

\begin{abstract}
A hybrid stochastic-deterministic approach for computing the second-order perturbative contribution $E^{(2)}$ within multireference perturbation theory (MRPT) is presented. The idea at the heart of our hybrid scheme-based on a reformulation of $E^{(2)}$ as a sum of elementary contributions associated with each determinant of the MR wave function-is to split $E^{(2)}$ into a stochastic and a deterministic part. During the simulation, the stochastic part is gradually reduced by dynamically increasing the deterministic part until one reaches the desired accuracy. In sharp contrast with a purely stochastic Monte Carlo scheme where the error decreases indefinitely as $t^{-1 / 2}$ (where $t$ is the computational time), the statistical error in our hybrid algorithm displays a polynomial decay $\sim t^{-n}$ with $n=3-4$ in the examples considered here. If desired, the calculation can be carried on until the stochastic part entirely vanishes. In that case, the exact result is obtained with no error bar and no noticeable computational overhead compared to the fully deterministic calculation. The method is illustrated on the $\mathrm{F}_{2}$ and $\mathrm{Cr}_{2}$ molecules. Even for the largest case corresponding to the $\mathrm{Cr}_{2}$ molecule treated with the cc-pVQZ basis set, very accurate results are obtained for $E^{(2)}$ for an active space of (28e, 1760) and a MR wave function including up to $2 \times 10^{7}$ determinants. Published by AIP Publishing. [http://dx.doi.org/10.1063/1.4992127]
\end{abstract}

\section{INTRODUCTION}

Multireference (MR) approaches are based upon the distinction between non-dynamical (or static) and dynamical correlation effects. Though such a clear-cut distinction is questionable, it is convenient to discriminate between the so-called static correlation effects emerging whenever the description of the molecular system using a single configuration breaks down (excited-states, transition-metal compounds, systems far from their equilibrium geometry, etc. $)^{1}$ and the dynamical correlation effects resulting from the short-range part of the electron-electron repulsion. ${ }^{2}$

To quantitatively establish this distinction, the Hamiltonian is decomposed as

$$
\hat{H}=\hat{H}^{(0)}+\hat{V},
$$

where the zeroth-order Hamiltonian $\hat{H}^{(0)}$ is chosen in conjunction with an MR wave function including the most chemically relevant configurations at the origin of static correlation effects, and

$$
\hat{V}=\hat{H}-\hat{H}^{(0)}
$$

is the residual part describing the bulk of dynamical correlation effects. The plethora of MR methods found in the literature results from the large freedom in choosing $\hat{H}^{(0)}$, and the fact that $\hat{V}$ may or may not be treated perturbatively. Among the non-perturbative approaches, let us cite the two most common ones, namely, the MR configuration interaction (MRCI) ${ }^{1,3,4}$ and the MR coupled cluster (MRCC) ${ }^{5-8}$ approaches. However,

\footnotetext{
a) Author to whom correspondence should be addressed: scemama@irsamc. ups-tlse.fr
}

because of their high computational cost, these methods are usually limited to systems of moderate size.

To overcome the computational burden associated with these methods-yet still capturing the main physical effectsa natural idea is to treat the potential as a perturbation, entering the realm of MR perturbation theories (MRPTs). Several flavors of MRPT exist depending on the choice of $\hat{H}^{(0)}$ (Epstein-Nesbet decomposition, ${ }^{9,10}$ Dyall Hamiltonian, ${ }^{11,12}$ Fink's partitioning, ${ }^{13,14}$ etc.). Among the most commonly used approaches, we have the CASPT2 ${ }^{15,16}$ and NEVPT2 $2^{11,12}$ methods. Regarding the construction of the zeroth-order part, CASSCF-type approaches are the most widely used schemes, ${ }^{17-19}$ but other methods, such as Complete Active Space Configuration Interaction (CASCI), selected CI (see Refs. 20 and 21 and the references therein), Full Configuration Interaction Quantum Monte Carlo (FCIQMC), ${ }^{22-24}$ or DMRG-type approaches ${ }^{25-27}$ can also be employed.

In this work, we shall consider MRPTs limited to the second order in perturbation (MRPT2). ${ }^{15}$ We address the important problem of calculating efficiently the second-order perturbative contribution $E^{(2)}$ in situations where standard calculations become challenging. Here, we suppose that the MR wave function has already been constructed by any method of choice.

Although the present method can be easily generalized to any externally decontracted MRPT approach (such as the recently introduced JM-MRPT2 method ${ }^{28}$ ), for the sake of simplicity, we shall restrict ourselves here to MR EpsteinNesbet perturbation theory. Extension to externally contracted methods, such as CASPT2 or NEVPT2, is less obviousalthough not impossible-since the excited contracted wave functions are non-orthogonal. 
The computational cost of MRPT2 can rapidly become unbearable when the number of electrons $N_{\mathrm{el}}$ and the number of one-electron basis functions $N_{\text {bas }}$ become large. The cost is indeed proportional to the number of reference determinants $N_{\text {det }}$ times the total number of singly and doubly excited determinants (scaling as $N_{\mathrm{el}}^{2} N_{\text {bas }}^{2}$ ). Because our main goal is to treat large, chemically relevant systems, the development of fast and accurate schemes for computing $E^{(2)}$ becomes paramount. Of course, in actual calculations, a trade-off must be found between the price to pay to build the MR wave function and the effort needed to evaluate $E^{(2)}$. Increasing $N_{\text {det }}$ (i.e., improving the MR wave function) may appear as the natural thing to do as the magnitude of $E^{(2)}$ decreases and the contribution of the neglected higher orders is made smaller. However, its computational price (proportional to $N_{\text {det }}$ ) increases stiffly, and the calculation becomes rapidly unfeasible. Of course, this balance is strongly dependent on the method used to generate the MR wave function and on the ability to compute rapidly and accurately $E^{(2)}$.

In this work, we present a simple and efficient Monte Carlo (MC) method for computing the second-order perturbative contribution $E^{(2)}$. For all the systems reported here, the reference space is constructed using the Configuration Interaction using a Perturbative Selection done Iteratively (CIPSI) method, ${ }^{20,21,29}$ a selected CI approach where important determinants are selected perturbatively. However, other variants of selected CI approaches or any other method for constructing the reference wave function may, of course, be used. Note that, in this study, the reported wall-clock times only refer to the computation of $E^{(2)}$, i.e., they do not take into account the preliminary calculation of the reference wave function.

A natural idea to evaluate $E^{(2)}$ with some targeted accuracy is to truncate the perturbational sum over excited determinants. However, since all the terms of the second-order sum have the same (negative) sign, the truncation will inevitably introduce a bias which is difficult to control. A way to circumvent this problem is to resort to a stochastic sampling of the various contributions. In this case, the systematic bias is removed at the price of introducing a statistical error. The key property is that this error can now be controlled, thanks to the central-limit theorem. However, in practice, to make the statistical average converge rapidly and to get statistical error small enough, care has to be taken in the way the statistical estimator is built and how the sampling is performed. The purpose of the present work is to propose a practical solution to this problem.

Note that the proposal of computing stochastically perturbative contributions is not new. In the context of secondorder Møller-Plesset (MP2) theory, where the reference Hamiltonian reduces to the Hartree-Fock Hamiltonian, Hirata and coworkers have proposed a MC scheme for calculating the MP2 correlation energy. ${ }^{30,31}$ However, we point out that this approach, based on a single-reference wave function, samples a 13-dimensional integral (in time and space) and has no direct relation with the present method. In a recent study, Sharma et al. ${ }^{32}$ address the very same problem of computing stochastically the second-order perturbative contribution of Epstein-Nesbet MRPT. Similarly to what is proposed here, $E^{(2)}$ is recast as a sum over contributions associated with each reference determinant, and contributions are stochastically sampled. However, the definition of the quantities to be averaged and the way the sampling is performed are totally different. Finally, let us mention the recent work of Jeanmairet et $a l .{ }^{33}$ addressing a similar problem in a different way. Within the framework of the recently proposed linear CC MRPT, it is shown that both the zeroth-order and firstorder wave functions can be sampled using a generalization of the FCIQMC approach. Here also, $E^{(2)}$ can be expressed as a stochastic average.

The present paper is organized as follows. In Sec. II, we report notations and basic definitions for MRPT2. Section III proposes an original reformulation of the secondorder contribution allowing an efficient MC sampling. The expression of the MC estimator is given, and a hybrid stochastic-deterministic approach greatly reducing the statistical fluctuations is presented. In Sec. IV, some illustrative applications for the $\mathrm{F}_{2}$ and $\mathrm{Cr}_{2}$ molecules are discussed. Finally, some concluding remarks are given in Sec. V.

\section{SECOND-ORDER MULTIREFERENCE PERTURBATION THEORY}

\section{A. Second-order energy contribution}

In MR Epstein-Nesbet perturbation theory, the reference Hamiltonian is chosen to be

$$
\hat{H}^{(0)}=E^{(0)}|\Psi\rangle\left\langle\Psi\left|+\sum_{\alpha \in \mathcal{A}} H_{\alpha \alpha}\right| \alpha\right\rangle\langle\alpha|,
$$

where $H_{\alpha \alpha}=\langle\alpha|\hat{H}| \alpha\rangle$ and

$$
|\Psi\rangle=\sum_{I \in \mathcal{D}} c_{I}|I\rangle
$$

is the reference wave function expressed as a sum of $N_{\text {det }}$ determinants belonging to the reference space

$$
\mathcal{D}=\left\{|I\rangle, I=1, \ldots, N_{\text {det }}\right\},
$$

and

$$
E^{(0)}=\frac{\langle\Psi|\hat{H}| \Psi\rangle}{\langle\Psi \mid \Psi\rangle}
$$

is the corresponding (variational) energy. The sum in Eq. (3) is over the set of determinants $|\alpha\rangle$ that do not belong to $\mathcal{D}$ but are connected to $\mathcal{D}$ via $\hat{H}$,

$$
\mathcal{A}=\left\{|\alpha\rangle \notin \mathcal{D} \wedge\left(\exists|I\rangle \in \mathcal{D} \mid H_{\alpha I} \neq 0\right)\right\} .
$$

Due to the two-body character of the interaction, the determinants $|\alpha\rangle$ are either singly or doubly excited with respect to (at least) one reference determinant. ${ }^{34}$ However, several reference determinants can be connected to the same $|\alpha\rangle$.

Using such notations, the second-order perturbative contribution is written as

$$
E^{(2)}=\sum_{\alpha \in \mathcal{A}} \frac{|\langle\alpha|\hat{H}| \Psi\rangle|^{2}}{\Delta E_{\alpha}},
$$

with $\Delta E_{\alpha}=E^{(0)}-H_{\alpha \alpha}$.

\section{B. Partition of $\mathcal{A}$}

The first step of the method-instrumental in the MC algorithm efficiency-is the partition of $\mathcal{A}$ into $N_{\text {det }}$ subsets $\mathcal{A}_{I}$ 
associated with each reference determinant $|I\rangle$,

$$
\mathcal{A}=\bigcup_{I=1}^{N_{\mathrm{det}}} \mathcal{A}_{I} \quad \text { with } \quad \mathcal{A}_{I} \cap \mathcal{A}_{J}=\emptyset \quad \text { if } \quad I \neq J .
$$

To define $\mathcal{A}_{I}$, the determinants $|I\rangle$ are first sorted in descending order according to the weight

$$
w_{I}=\frac{c_{I}^{2}}{\langle\Psi \mid \Psi\rangle} .
$$

The partition of $\mathcal{A}$ starts with $\mathcal{A}_{1}$ defined as the set of determinants $|\alpha\rangle \in \mathcal{A}$ connected to the first reference determinant (i.e., $I=1$ ). Then, $\mathcal{A}_{2}$ is constructed as the set of determinants of $\mathcal{A}$ connected to the determinant corresponding to $I=2$, but not belonging to $\mathcal{A}_{1}$. The process is carried on up to the last determinant. This partition is schematically illustrated in Fig. 1. Mathematically, it can be written as

$$
\mathcal{A}_{I}=\left\{|\alpha\rangle \in \mathcal{A} \mid H_{\alpha I} \neq 0 \wedge\left(\forall J<I,|\alpha\rangle \notin \mathcal{A}_{J}\right)\right\} .
$$

Because of the way they are constructed, the size of $\mathcal{A}_{I}$ is expected to decrease rapidly as a function of $I$, except for a possible transient regime for very small $I$.

A key point in the construction of the partition of $\mathcal{A}$ is to avoid both the computation of redundant contributions and the storage of unnecessary intermediates. First, when a determinant $|\alpha\rangle$ is generated by applying a single or double excitation operator to a reference determinant $|I\rangle$, one has to check that $|\alpha\rangle$ does not belong to $\mathcal{D}$. If the reference determinants are stored in a hash table, the presence of $|\alpha\rangle$ in $\mathcal{D}$ can be checked in constant time. Next, one has to know if $|\alpha\rangle$ has already been generated via another reference determinant $|J\rangle$. To do so, one must compute the number of holes and particles between $|\alpha\rangle$ and each determinant preceding $|I\rangle$ in $\mathcal{D}$. As soon as an excitation degree lower than 3 is found, the search can be aborted since the contribution is known to have been considered before. In the worst-case scenario, this step scales as $\mathcal{O}\left(N_{\text {det }}\right)$, and the prefactor is very small since finding the excitation degree between two determinants can be performed in less than 20 CPU cycles $^{35}$ (comparable to a floating-point division). Furthermore, the asymptotic scaling can be further reduced by

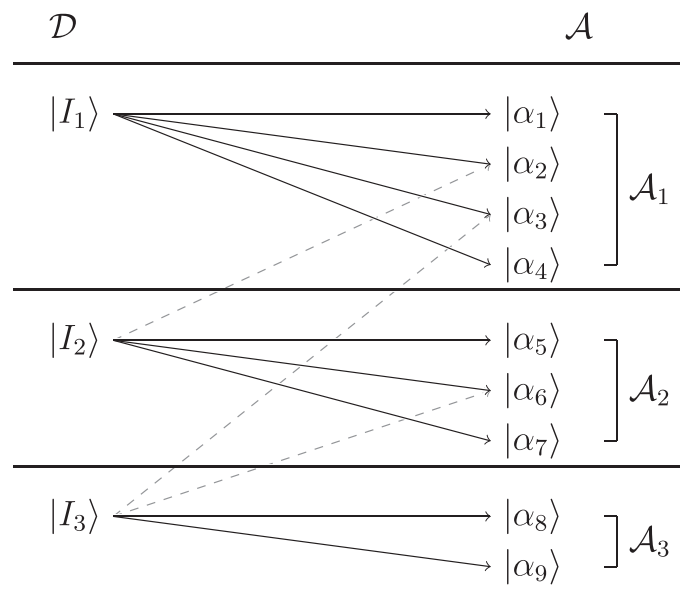

FIG. 1. Iterative construction of the subsets $\mathcal{A}_{I}$. Arrows indicate a non-zero matrix element $H_{I \alpha}=\langle I|\hat{H}| \alpha\rangle$. Solid arrows: the determinant $|\alpha\rangle$ is accepted as a member of the subset $\mathcal{A}_{I}$. Dotted arrows: the determinant $|\alpha\rangle$ already belongs to a previous subset $\mathcal{A}_{J<I}$ and is therefore not incorporated into $\mathcal{A}_{I}$.
TABLE I. Convergence of $E^{(2)}$ for the $\mathrm{Cr}_{2}$ molecule with bond length 1.68 $\AA$ as a function of the wall-clock time for various basis sets (800 CPU cores).

\begin{tabular}{lcc}
\hline \hline Basis & $E^{(2)}$ & Wall-clock time \\
\hline cc-pVDZ & $-0.0683(1)$ & $14 \mathrm{~min}$ \\
& $-0.06836(1)$ & $55 \mathrm{~min}$ \\
& $-0.068361(1)$ & $2.4 \mathrm{~h}$ \\
& -0.068360604 & $3 \mathrm{~h}$ \\
\hline cc-pVTZ & $-0.1244(5)$ & $19 \mathrm{~min}$ \\
& $-0.1247(1)$ & $58 \mathrm{~min}$ \\
& $-0.12463(1)$ & $3.5 \mathrm{~h}$ \\
& $-0.124642(1)$ & $8.7 \mathrm{~h}$ \\
& $\ldots$ & $\sim 15 \mathrm{~h}(\mathrm{estimated})$ \\
\hline cc-pVQZ & $-0.1558(5)$ & $56 \mathrm{~min}$ \\
& $-0.1559(1)$ & $2.5 \mathrm{~h}$ \\
& $-0.15595(1)$ & $9.0 \mathrm{~h}$ \\
& $-0.155952(1)$ & $18.5 \mathrm{~h}$ \\
& $\ldots$ & $29 \mathrm{~h}(\mathrm{estimated})$ \\
\hline \hline
\end{tabular}

sorting the determinants in groups with the same spin string. Indeed, one only has to probe determinants $|J\rangle$ that are no more than quadruply excited with respect to $|I\rangle$, and if the search is restricted to groups with the same spin-up string, the asymptotic scaling reduces to $\mathcal{O}\left(\sqrt{N_{\mathrm{det}}}\right)$. To provide a quantitative illustration of the computational effort associated with the construction of the partitioning, using $2 \times 10^{7}$ determinants (as in the case of $\mathrm{Cr}_{2}$ presented below), this preliminary step is negligible: on a single $2.7 \mathrm{GHz}$ core, the calculation takes 20 cycles $\times N_{\text {det }}^{3 / 2} /\left(2.7 \times 10^{9}\right.$ cycles $\left./ \mathrm{s}\right) \sim 663 \mathrm{~s}(\mathrm{CPU}$ time), while the total execution time (wall-clock time) of the entire run ranges from $14 \mathrm{~min}$ to $18.5 \mathrm{~h}$ using 800 cores (see Table I).

\section{Partition of $E^{(2)}$}

Thanks to the partition of $\mathcal{A}$ [see Eq. (11)], the sum (8) can be decomposed into a sum over the reference determinants $|I\rangle$,

$$
E^{(2)}=\sum_{I=1}^{N_{\mathrm{det}}} e_{I}
$$

where

$$
e_{I}=\sum_{\alpha \in \mathcal{A}_{I}} \frac{|\langle\alpha|\hat{H}| \Psi\rangle|^{2}}{\Delta E_{\alpha}} .
$$

Moreover, noticing that by construction, the determinants $|\alpha\rangle$ belonging to $\mathcal{A}_{I}$ are not connected to the part of the reference function expanded over the preceding reference determinants; we have

$$
e_{I}=\sum_{\alpha \in \mathcal{A}_{I}} \frac{\left|\left\langle\alpha|\hat{H}| \Psi_{I}\right\rangle\right|^{2}}{\Delta E_{\alpha}}
$$

where

$$
\left|\Psi_{I}\right\rangle=\sum_{J=I}^{N_{\mathrm{det}}} c_{J}|J\rangle
$$

is a truncated reference wave function. Our final working expression for the second-order contribution $E^{(2)}$ is thus 


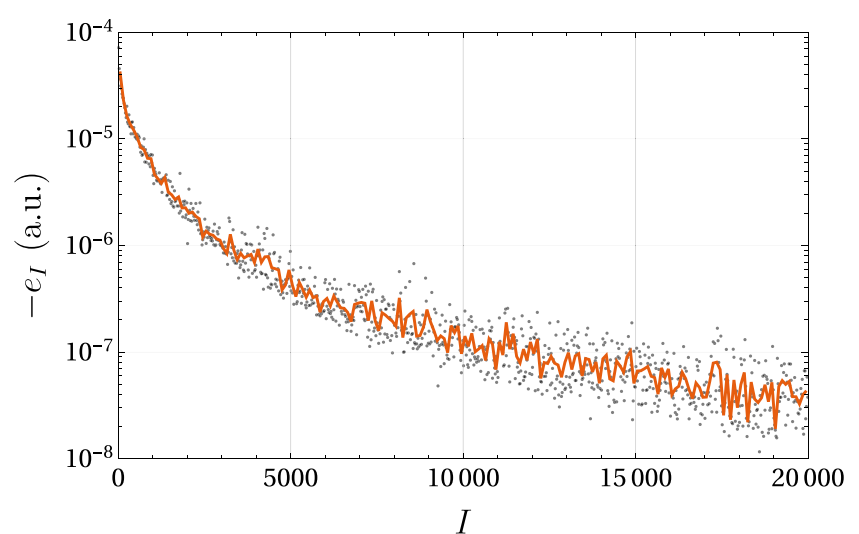

FIG. 2. $-e_{I}$ as a function of $I$ for the first 20000 determinants selected by the CIPSI method for the $\mathrm{F}_{2}$ molecule at equilibrium geometry with the cc-pVQZ basis set. The two sets of data are obtained by averaging either by groups of 20 (point cloud) or 100 (solid line) values.

written as

$$
E^{(2)}=\sum_{I=1}^{N_{\mathrm{det}}} e_{I}=\sum_{I=1}^{N_{\mathrm{det}}} \sum_{\alpha \in \mathcal{A}_{I}} \frac{\left|\left\langle\alpha|\hat{H}| \Psi_{I}\right\rangle\right|^{2}}{\Delta E_{\alpha}} .
$$

A key property at the origin of the efficiency of the MC simulations presented below is that $e_{I}$ 's take their largest values at very small $I$. Then, they decay very rapidly as $I$ increases.

This important property is illustrated in Fig. 2. The data have been obtained for the $\mathrm{F}_{2}$ molecule at the equilibrium bond length of $R_{\mathrm{F}-\mathrm{F}}=1.4119 \AA$ using Dunning's cc-pVQZ basis set. ${ }^{36}$ The multideterminant reference space is built by selecting determinants using the CIPSI algorithm. Figure 2 displays $e_{I}$ 's for the first 20000 selected determinants. As one can see, $e_{I}$ 's decay very rapidly with $I$. Of course, at the scale of individual determinants, there is no guarantee of a strictly monotonic decay, and it is indeed what we observe. By averaging groups of successive $e_{I}$ 's, the curve can be smoothed out. The two data sets presented in Fig. 2 have been obtained by averaging either by groups of 20 (point cloud) or 100 (solid line) values.

It is important to note that the rapid decay of $e_{I}$ 's is a direct consequence of the way we have chosen to decompose $\mathcal{A}$. To be more precise, we note that in Eq. (14), the decay has three different origins:

- the number of determinants involved in the sum over $|\alpha\rangle$ decreases as a function of $I$;

- the excitation energies $\Delta E_{\alpha}$ increase with $I$;

- the norm of the truncated wave function $\Psi_{I}$ decreases rapidly (as $c_{I}^{2}$ ) when $I$ increases.

In addition, as a consequence of the first point, we note that the computation of $e_{I}$ becomes faster when $I$ increases.

\section{MONTE CARLO METHOD}

\section{A. Monte Carlo estimator}

To get an expression of $E^{(2)}$ suitable for MC simulations, the second-order contribution is recast as

$$
E^{(2)}=\sum_{I=1}^{N_{\mathrm{det}}} p_{I}\left(\frac{e_{I}}{p_{I}}\right)
$$

and is thus rewritten as the following MC estimator:

$$
E^{(2)}=\left\langle\frac{e_{I}}{p_{I}}\right\rangle_{p_{I}}
$$

Here, $p_{I}$ is an arbitrary probability distribution. The optimal choice for $p_{I}$ is given by the zero-variance condition, i.e.,

$$
p_{I}^{\mathrm{opt}}=\frac{e_{I}}{E^{(2)}}
$$

Note that $e_{I}$ and $E^{(2)}$ being both negative, the probability distribution $p_{I}$ is positive, as it should be.

To build a reasonable approximation of $p_{I}$, we note that the magnitude of $e_{I}$, as expressed in Eq. (14), is essentially given by the norm of the truncated wave function $\Psi_{I}$ [see Eq. (15)]. Thus, a natural choice for the probability distribution is

$$
p_{I}=\frac{\left\langle\Psi_{I} \mid \Psi_{I}\right\rangle}{\sum_{J=1}^{N_{\mathrm{det}}}\left\langle\Psi_{J} \mid \Psi_{J}\right\rangle}=\frac{\sum_{J=I}^{N_{\mathrm{det}}} c_{J}^{2}}{\sum_{J=1}^{N_{\mathrm{det}}} \sum_{K=J}^{N_{\mathrm{det}}} c_{K}^{2}} .
$$

In our simulations, we have observed that summing totally or partially the squared coefficients in the numerator does not change significantly the statistical fluctuations. As a consequence, we restrict the summation in Eq. (20) to the leading term, i.e.,

$$
p_{I}=\frac{c_{I}^{2}}{\sum_{J=1}^{N_{\mathrm{det}}} c_{J}^{2}}=w_{I}
$$

Let us emphasize that performing a MC simulation in the $e_{I}$ space is highly beneficial since the number of $e_{I}$ is always small enough to make them all fit in memory. Hence, one can follow the so-called lazy evaluation strategy: ${ }^{37}$ the value of $e_{I}$ is computed only once when needed for the first time, and its value is then stored. If the same $e_{I}$ is requested later, the stored value will be returned.

\section{B. Improved Monte Carlo sampling}

The stochastic calculation of $E^{(2)}$, Eq. (18), can be done in a standard way by sampling the probability distribution and averaging the successive values of $e_{I} / p_{I}$. In practice, the sampling can be realized by drawing, at each MC step, a uniform random number $u \in[0,1]$ and selecting the determinant $|I\rangle$ verifying

$$
R(I-1) \leq u \leq R(I),
$$

where $R$ is the cumulative distribution function of the probability distribution defined as

$$
R(I)=\sum_{J=1}^{I} p_{J}
$$

with $R(0)=0$.

At this stage, it is useful to take advantage of the fact that, thanks to the way $e_{I}$ 's have been constructed, the quantity to be averaged, $e_{I} / p_{I}$, is a slowly varying function of $I$ (providing that the small-scale fluctuations present at the level of individual determinants have been averaged out). This property, which is well illustrated by Fig. 2 , is shared by $p_{I} \sim c_{I}^{2}$, hence by the ratio $e_{I} / p_{I}$. Thus, an efficient way to reduce the statistical fluctuations consists in sampling piece-wisely $\mathcal{D}$ by decomposing it into subdomains where the integrand is a slowly varying function [see the justification of this statement after Eq. (30)]. 
To implement this idea, the interval $[0,1]$ is divided into $M$ equally spaced intervals $\mathcal{U}_{k}$ and a "comb" of correlated random numbers

$$
u_{k}=\frac{k-1+u}{M}, \quad \text { for } k=1, \ldots, M,
$$

covering uniformly $[0,1]$ is created (where $u$ is a single uniform random number). At each MC step, a $M$-tuple of determinants $\left(I_{1}, I_{2}, \ldots, I_{M}\right)$ verifying

$$
R\left(I_{k}-1\right) \leq u_{k} \leq R\left(I_{k}\right), \quad \text { for } k=1, \ldots, M
$$

is drawn.

Defining $\mathcal{D}_{k}$ as the subset of determinants $\left|I_{k}\right\rangle$ satisfying $R\left(I_{k}\right) \in \mathcal{U}_{k}$, we introduce the following partition:

$$
\mathcal{D}=\bigcup_{k=1}^{M} \mathcal{D}_{k} \quad \text { with } \quad \mathcal{D}_{k} \cap \mathcal{D}_{l}=\emptyset, \forall k \neq l
$$

and express $E^{(2)}$ as a sum of $M$ contributions associated with each $\mathcal{D}_{k}$,

$$
E^{(2)}=\sum_{k=1}^{M} \sum_{I_{k} \in \mathcal{D}_{k}} e_{I_{k}} .
$$

Using the process described above [Eqs. (24) and (25)], the second-order energy can be rewritten as the following MC estimator:

$$
E^{(2)}=\left\langle\frac{1}{M} \sum_{k=1}^{M} \frac{e_{I_{k}}}{p_{I_{k}}}\right\rangle_{p\left(I_{1}, \ldots, I_{M}\right)},
$$

where $p\left(I_{1}, \ldots, I_{M}\right)$ denotes the normalized probability distribution corresponding to Eqs. (24) and (25). Equation (28) follows from the fact that, by construction, $p_{I_{k}}$ is the $k$ th marginal distribution of $p\left(I_{1}, \ldots, I_{M}\right)$,

$$
\sum_{I_{1}} \cdots \sum_{I_{k-1}} \sum_{I_{k+1}} \cdots \sum_{I_{M}} p\left(I_{1}, \ldots, I_{M}\right)=M p_{I_{k}},
$$

with

$$
\sum_{I_{k} \in \mathcal{D}_{k}} p_{I_{k}}=\frac{1}{M}
$$

By drawing determinants on separate subsets $\mathcal{D}_{k}$, the sum to be averaged in Eq. (28) is expected to fluctuate less than the very same sum computed by independently drawing determinants over $\mathcal{D}$. This remarkable property can be explained as follows. For large $M$, the fluctuations of the sum based on independent drawings behave as in any MC scheme, i.e., as $M^{-1 / 2}$. Using a comb covering evenly (with weight $p_{I}$ ) the determinant space, the situation is different since the sum can now be seen as a Riemann sum over $\mathcal{D}$ with a residual error behaving as $M^{-1}$. As a consequence, the overall reduction in statistical noise resulting from the use of the comb is expected to be of the order of $\sqrt{M}$. We emphasize that such an attractive feature is only observed because $e_{I} / p_{I}$ is a slowly varying function of I (as mentioned above). In the opposite case, the gain would vanish. In the application on the $F_{2}$ molecule presented below (see Fig. 5), the numerical results confirm this: a decrease of about one order of magnitude in statistical error is obtained when using $M=100$. Note that using a comb reduces the estimator's variance but does not change the typical inverse square root behavior of the statistical error with respect to the number of MC steps.
Note that Eq. (26) is actually not correct when some determinants (first and/or last determinant of a given subset) belong to more than one subset. Thus, special care has to be taken for determinants at the boundary of two subsets, but this difficulty can be easily circumvented by formally duplicating each of these determinants into copies with suitable weights.

\section{Hybrid stochastic-deterministic scheme}

In practice, because the first few determinants are responsible for the most significant contribution in Eq. (17), it is advantageous not to sample the entire reference space but to remove from the stochastic sampling the leading determinants. Consequently, $E^{(2)}$ is split into a deterministic $E_{\mathrm{D}}^{(2)}$ and a stochastic $E_{\mathrm{S}}^{(2)}$ component, such as

$$
\begin{aligned}
E^{(2)} & =E_{\mathrm{D}}^{(2)}+E_{\mathrm{S}}^{(2)} \\
& =\sum_{J \in \mathcal{D}_{\mathrm{D}}} e_{J}+\left\langle\frac{1}{M} \sum_{k=1}^{M} \frac{e_{I_{k}}}{p_{I_{k}}}\right\rangle_{p\left(I_{1}, \ldots, I_{M}\right)},
\end{aligned}
$$

where $\mathcal{D}_{\mathrm{D}}$ is the set of determinants in the deterministic space, and $\mathcal{D}_{\mathrm{S}}=\mathcal{D} \backslash \mathcal{D}_{\mathrm{D}}$ is its stochastic counterpart.

At a given point of the simulation, some determinants have been drawn, and some have not. If we keep track of the list of the drawn determinants, we can check periodically, for each $\mathcal{D}_{k}$, whether or not all elements have been drawn at least once. If that is the case, the full set of determinants is moved to $\mathcal{D}_{\mathrm{D}}$ and the corresponding contribution $\sum_{I_{k}} e_{I_{k}}$ is added to $E_{\mathrm{D}}^{(2)}$. The statistical average and error bar are then updated accordingly. The expression of the $E^{(2)}$ estimator is now time-dependent and, at the $m$ th MC step, the deterministic part is given by

$$
E_{\mathrm{D}}^{(2)}(m)=\sum_{k=1}^{M} \Theta_{k}(m) \sum_{I_{k}} e_{I_{k}},
$$

where

$$
\Theta_{k}(m)= \begin{cases}1, & \text { if } \mathcal{D}_{k} \subset \mathcal{D}_{\mathrm{D}} \text { at step } m, \\ 0, & \text { otherwise. }\end{cases}
$$

On the other hand, the stochastic part is now given by

$$
E_{\mathrm{S}}^{(2)}(m)=\frac{1}{M} \sum_{k=1}^{M}\left[1-\Theta_{k}(m)\right] \sum_{I_{k} \in \mathcal{D}_{k}} w_{I_{k}}^{(m)} \frac{e_{I_{k}}}{p_{I_{k}}},
$$

where

$$
w_{I_{k}}^{(m)}=\frac{n_{I_{k}}^{(m)}}{\sum_{J_{k} \in \mathcal{D}_{k}} n_{J_{k}}^{(m)}},
$$

and $n_{I_{k}}^{(m)}$ denotes the number of times the determinant $I_{k}$ has been drawn at iteration $m$.

If desired, the calculation can be carried on until the stochastic part entirely vanishes. In that case, all the determinants are in $\mathcal{D}_{\mathrm{D}}$, and the exact value of $E^{(2)}$ is obtained with zero statistical fluctuations.

Finally, to make sure that a given set $\mathcal{D}_{k}$ does not stay in the stochastic part because a very small number of its determinants have not been drawn, we have implemented an additional step as follows. At each MC iteration (where a new comb is created), the contribution $e_{I}$ of the first not-yet-sampled determinant (i.e., corresponding to the smallest $I$ value in the sorted 
determinant list) is calculated and stored. By doing this, the convergence of the hybrid stochastic-deterministic estimator is significantly improved. Moreover, after $N_{\text {det }}$ MC steps, it is now guaranteed that the exact deterministic value is reached.

\section{Upper bound on the computational time}

In the present method, the vast majority of the computational time is spent calculating $e_{I}$ 's. A crucial point which makes the algorithm particularly efficient is the lazy evaluation of these quantities. This implies that, in practice, the stochastic calculation will never be longer than the time needed to compute all the individual $e_{I}$ 's (i.e., the time necessary to complete the fully deterministic calculation) due to the negligible time required by the $\mathrm{MC}$ sampling (drawing $100 \times 10^{6}$ random numbers takes less than $3 \mathrm{~s}$ on a single CPU core).

Finally, it is noteworthy that the final expression of $E^{(2)}$ can be very easily decomposed into (strictly) independent calculations. The algorithm presented here is thus embarrassingly parallel (see Sec. IV C).

\section{NUMERICAL TESTS}

The present algorithm has been implemented in our Quantum Package code. ${ }^{38}$ The perturbatively selected CI algorithm CIPSI, ${ }^{20,29}$ as described in Ref. 21 , is used to build the multideterminant reference space. In all the calculations performed in this section, we have chosen to use a comb with $M=100$. All the simulations were performed on the Curie supercomputer (TGCC/CEA/GENCI) where each node is a dual socket Xeon E5-2680 at $2.70 \mathrm{GHz}$ with $64 \mathrm{~GB}$ of RAM, interconnected with an Infiniband QDR network.

\section{A. $F_{2}$ molecule}

As a first illustrative example, we consider the calculation of $E^{(2)}$ for the $\mathrm{F}_{2}$ molecule in its ${ }^{1} \Sigma_{g}^{+}$electronic ground state at equilibrium geometry. The two $1 s$ core electrons are kept frozen, and Dunning's cc-pVQZ basis set is used. The Hilbert space is built by distributing the 14 active electrons within the 108 non-frozen molecular orbitals for a total of more than $10^{20}$ determinants.

Despite the huge size of the Hilbert space, the selected CI approach is able to reach the full CI (FCI) limit with a very good accuracy. The convergence of the variational energy $E^{(0)}$ and that of the total energy (given by the sum of the variational and second-order contribution $E^{(0)}+E^{(2)}$ ) with respect to the

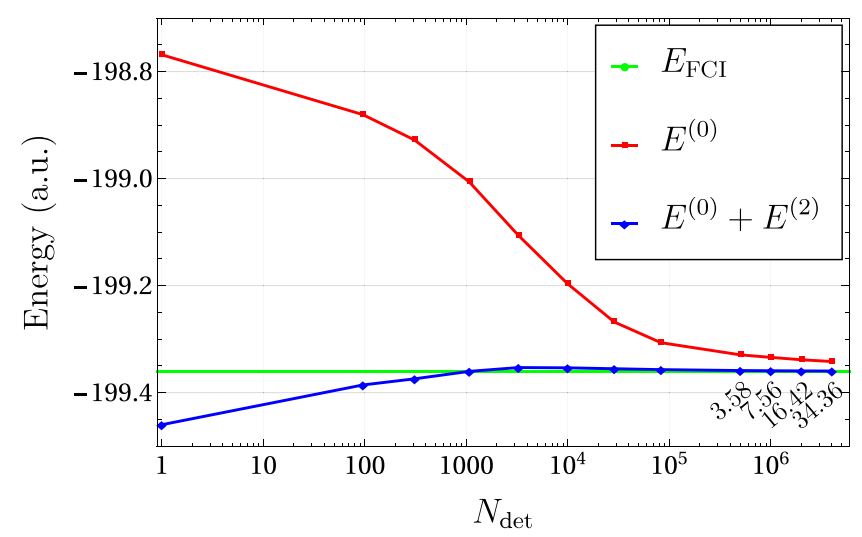

FIG. 3. $F_{2}$ molecule at equilibrium geometry. Convergence of the variational energy $E^{(0)}$ (red curve) as a function of the number of selected determinants $N_{\text {det }}$ obtained with the CIPSI method and the cc-pVQZ basis set. The blue curve is obtained by adding the second-order energy contribution $E^{(2)}$ to the variational one $E^{(0)}$. The full CI $(\mathrm{FCI})$ value (green curve) is reported as a reference. The wall-clock time (in minutes) needed to compute $E^{(2)}$ for various values of $N_{\text {det }}$ is also reported (black numbers underneath the blue curve).

number of selected determinants are presented in Fig. 3. The maximum number of determinants we have selected is $4 \times 10^{6}$. For this value, $E^{(0)}$ is not converged but is already a reasonable approximation to the FCI energy with an error of about $18 \mathrm{~m} E_{\mathrm{h}}$. In sharp contrast, the total energy including the second-order correction converges very rapidly: millihartree accuracy is reached with about $2 \times 10^{6}$ determinants. For $N_{\text {det }}=4 \times 10^{6}$, the best value obtained is -199.3594 a.u., in quantitative agreement with the estimated FCI value of -199.3598 (2) a.u. obtained by Cleland $e$ t al. with FCIQMC. ${ }^{23}$

For this system and the maximum number of selected determinants considered, it is actually possible to calculate exactly $E^{(2)}$ by explicit evaluation of the entire sum (deterministic method). The corresponding wall-clock times (in minutes) using 50 nodes (800 cores) are reported directly in Fig. 3. For $N_{\text {det }}=10^{4}$, the calculation takes a few seconds, while for the largest number of $N_{\text {det }}=4 \times 10^{6}$ about $35 \mathrm{~min}$ are needed.

We now consider the hybrid stochastic-deterministic evaluation of $E^{(2)}$. The left graph of Fig. 4 shows the evolution of $E^{(2)}$ as a function of the wall-clock time (in minutes). Data are given for the cc-pVQZ basis and $N_{\text {det }}=4 \times 10^{6}$. Similar curves are obtained with the two other basis sets. As one can see, the rate of convergence of the error is striking, and eventually, the exact value is obtained with very small fluctuations. If chemical accuracy is targeted (error of roughly $1 \mathrm{~m} E_{\mathrm{h}}$ ),

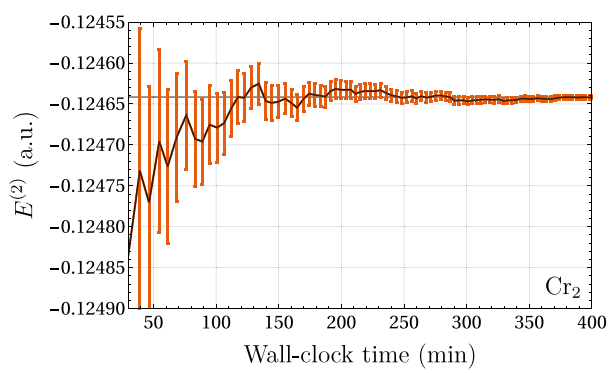

FIG. 4. Convergence of $E^{(2)}$ as a function of the wall-clock time for the $\mathrm{F}_{2}$ molecule (left) with $N_{\text {det }}=4 \times 10^{6}$ (cc-pVQZ basis set) and the Cr 2 molecule (right) with $N_{\text {det }}=2 \times 10^{7}$ (cc-pVTZ basis set). Both graphs are obtained with $800 \mathrm{CPU}$ cores. The grey line corresponds to the exact (deterministic) value for $\mathrm{F}_{2}$ and to the value with the lowest statistical error for $\mathrm{Cr}_{2}$. The error bars correspond to one standard deviation. 


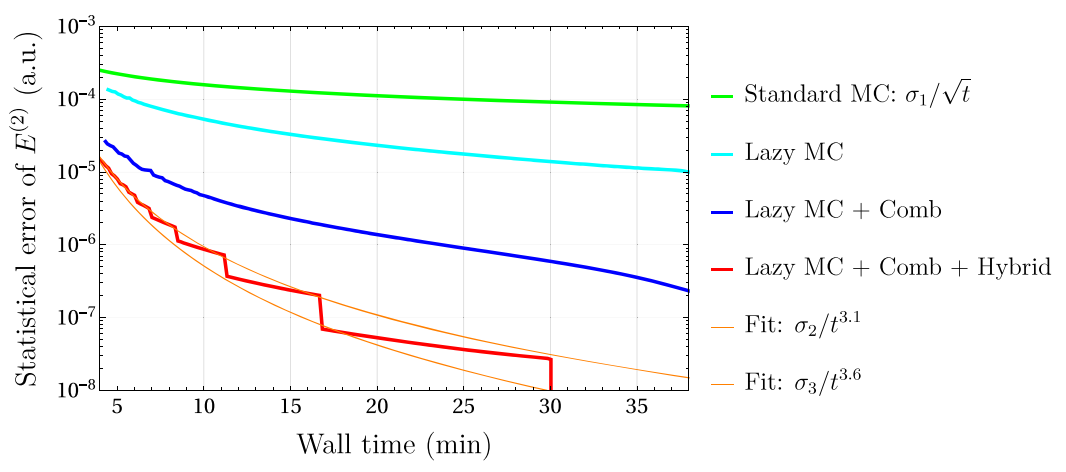

FIG. 5. Statistical error of $E^{(2)}$ as a function of the wallclock time for the $\mathrm{F}_{2}$ molecule obtained with the cc-pVQZ basis and $N_{\text {det }}=4 \times 10^{6}$ with different schemes.
3 min are needed using 800 cores. This value has to be compared with the $\sim 35$ min needed to evaluate the exact value (see Fig. 3).

To have a better look at the fluctuations, the statistical error as a function of the wall-clock time is reported in Fig. 5. We have reported four curves to show the effects of the different strategies used in our algorithm. The first one (in green) is the curve one would typically obtain using a standard MC algorithm where the contributions are always recomputed (no lazy evaluation). Note that, for this particular curve, we have not performed the calculation, but we have plotted an arbitrary $\sigma_{1} / \sqrt{t}$ curve to illustrate its decay rate. The light blue curve is obtained using the MC estimator proposed in Sec. III A. The slope is steeper than that for the standard MC scheme, thanks to the lazy evaluation strategy. The introduction of the comb (Sec. III B) reduces the statistical error by an order of magnitude and produces the dark blue curve. Finally, incorporating the hybrid deterministic/stochastic scheme (Sec. III C) yields the red curve.

Quite remarkably, the overall convergence of the red curve is extremely rapid. Because of the irregular convergence, it is not easy to extract the exact mathematical form of the decay. However, it is clear that a typical polynomial decay is observed. Fitting the curve of the hybrid scheme gives a decrease of the error bar between $t^{-3.1}$ and $t^{-3.6}$, which is significantly faster than the $t^{-1 / 2}$ behavior of the standard MC algorithm. Note also that some discontinuities in the statistical error are regularly observed. Such sudden drops occur each time a subset $\mathcal{D}_{k}$ is entirely filled and its contribution is transferred to the deterministic part. Comparison with the standard MC algorithm illustrates that obtaining an arbitrary accuracy with a standard MC sampling can rapidly become prohibitively expensive. Most importantly, the wall-clock time would rapidly become larger than the time required to compute exactly (i.e., deterministically) $E^{(2)}$, which is not the case with the here-proposed method.

\section{B. $\mathrm{Cr}_{2}$ molecule}

We now consider the challenging example of the $\mathrm{Cr}_{2}$ molecule in its ${ }^{1} \Sigma_{\mathrm{g}}^{+}$ground state. The internuclear distance is chosen to be close to its experimental equilibrium geometry, i.e., $R_{\mathrm{Cr}-\mathrm{Cr}}=1.68 \AA$. Full-valence calculations including 28 active electrons (two frozen neon cores) are performed. The cc-pVDZ, TZ, and QZ basis sets ${ }^{39}$ are employed, and the associated active spaces corresponding to $(28 \mathrm{e}, 76 \mathrm{o}),(28 \mathrm{e}$, $126 \mathrm{o})$, and (28e, 176o) include more than $10^{29}, 10^{36}$, and $10^{42}$ determinants, respectively. For all the basis sets, the molecular orbitals (MOs) were obtained with the GAMESS ${ }^{40}$ program using a CASSCF calculation with 12 electrons in 12 orbitals, and $2 \times 10^{7}$ determinants were selected in the FCI space with the CIPSI algorithm implemented in Quantum Package. In the cc-pVQZ basis set, we had to remove the $h$ functions of the basis set since the version of GAMESS we used (prior to 2013) does not handle the corresponding two-electron integrals.

The right graph of Fig. 4 shows the convergence of $E^{(2)}$ as a function of the wall-clock time for the cc-pVTZ basis set and $N_{\text {det }}=2 \times 10^{7}$. Again, similar curves are obtained with the two other basis sets. Similarly to $F_{2}$, the convergence is remarkably fast with a steep decrease of the statistical error with respect to the wall-clock time (for quantitative results, see Table I). Note that the maximum energy range in the right graph of Fig. 4 is only $0.35 \mathrm{~m} E_{\mathrm{h}}$.

Table II reports the quantitative results obtained with the three basis sets. One can observe that very accurate results for $E^{(2)}$ can be obtained even with the largest QZ basis set. For the three basis sets, the statistical error obtained is $10^{-6} E_{\mathrm{h}}$. However, it is clear that in practical applications, we do not need such high level of accuracy as the finite-size basis effects as well as the high-order perturbative contributions are much larger. If, more reasonably, we target an accuracy of about $0.1 \mathrm{~m} E_{\mathrm{h}}$, we see in Table I that the wall-clock time needed is

TABLE II. Variational ground-state energy $E^{(0)}$ and second-order contribution $E^{(2)}$ of the $\mathrm{Cr}_{2}$ molecule with bond length $1.68 \AA$ computed with various basis sets. For all basis sets, the reference is composed of $2 \times 10^{7}$ determinants selected in the valence FCI space ( 28 electrons).

\begin{tabular}{lccccr}
\hline \hline Reference & Basis & Active space & $E^{(0)}$ & $E^{(2)}$ & $E^{(0)}+E^{(2)}$ \\
\hline CIPSI & cc-pVDZ & $(28 \mathrm{e}, 760)$ & -2087.2278833 & $-0.068334(1)$ & $-2087.296217(1)$ \\
& cc-pVTZ & $(28 \mathrm{e}, 1260)$ & -2087.4497817 & $-0.124676(1)$ & $-2087.574423(1)$ \\
& cc-pVQZ & $(28 \mathrm{e}, 1760)$ & -2087.5133733 & $-0.155957(1)$ & $-2087.669330(1)$ \\
\hline \hline
\end{tabular}




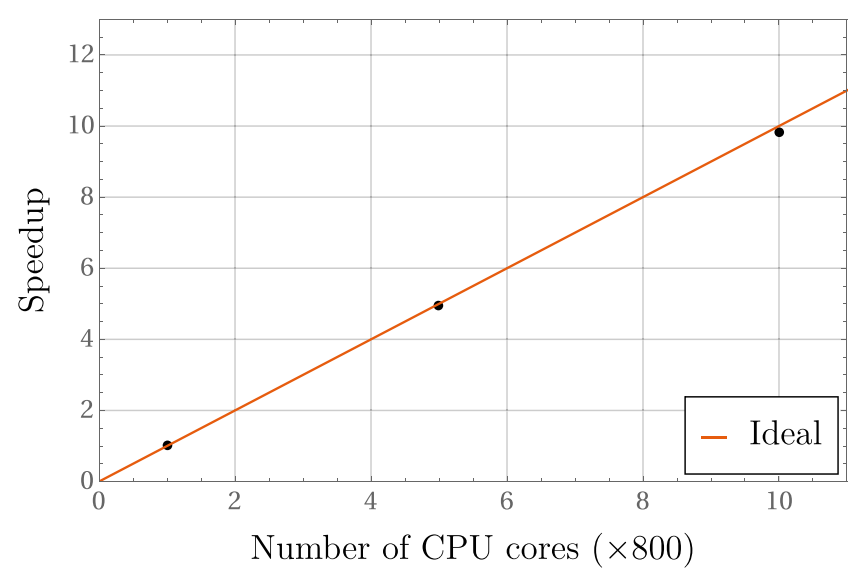

FIG. 6. Parallel speedup of our implementation using 800,4000 , and 8000 cores. The reference is the 800-core run.

about $14 \mathrm{~min}, 1 \mathrm{~h}$, and $2.5 \mathrm{~h}$ with $800 \mathrm{CPU}$ cores for the DZ, TZ, and QZ basis sets, respectively. Finally, we note that, in contrast with $\mathrm{F}_{2}$, the absolute value of $E^{(2)}$ remains large even when relatively large MR wave functions are employed. This result clearly reflects the difficulty in treating accurately $\mathrm{Cr}_{2}$. We postpone to a forthcoming paper the detailed analysis of this system and the calculation of the entire potential energy curve.

\section{Parallel speedup}

To measure the parallel speedup of the present implementation of our algorithm, we have measured the wall-clock time needed to reach a target statistical error of $10^{-6}$ a.u. with 800,4000 , and 8000 cores $(50,250$, and 500 nodes) using the $\mathrm{Cr}_{2} / \mathrm{cc}-\mathrm{pVQZ}$ wave function with $N_{\mathrm{det}}=2 \times 10^{7}$. The speedup is calculated using the 800-core run as the reference, and the results are shown in Fig. 6. Going from 800 to 4000 cores gives a speedup of 4.95 , and the 8000-core run exhibits a speedup of 9.82 . These values are extremely close to the ideal values of 5 and 10 . Therefore, we believe that this method is a good candidate for running on exascale machines in a near future.

\section{CONCLUSIONS}

In this work, a hybrid stochastic-deterministic algorithm to compute the second-order energy contribution $E^{(2)}$ within the Epstein-Nesbet MRPT has been introduced. Two main ideas are at the heart of the method. First, the reformulation of the standard expression of $E^{(2)}$, Eq. (8), into Eq. (16). Thanks to the unique property of the elementary contributions $e_{I}$; the latter expression turns out to be particularly well-suited for low-variance MC calculations. The second idea, which greatly enhances the convergence of the calculation, is to decompose $E^{(2)}$ as a sum of a deterministic and a stochastic part, the deterministic part being dynamically updated during the calculation.

We have observed that the size of the stochastic part (as well as the statistical error) decays in time with a polynomial behavior. If desired, the calculation can be carried on until the stochastic part entirely vanishes. In that case, the exact (deterministic) result is obtained with no error bar and no noticeable computational overhead compared to the fully deterministic calculation. Such a remarkable result is in sharp contrast with standard MC calculations where the statistical error decreases indefinitely as the inverse square root of the simulation time.

The numerical applications presented for the $\mathrm{F}_{2}$ and $\mathrm{Cr}_{2}$ molecules illustrate the great efficiency of the method. The largest calculation on $\mathrm{Cr}_{2}$ (cc-pVQZ basis set) has an active space of (28e, 176o), corresponding to a Hilbert space consisting of approximately $10^{42}$ determinants and a multireference wave function containing $2 \times 10^{7}$ determinants. Even in this extreme case, $E^{(2)}$ can easily be calculated with submillihartree accuracy using a fully and massively parallel version of the algorithm.

As a final comment, we would like to mention that, although we have only considered two illustrative examples in the present manuscript, our method has been shown to be highly successful in all the cases we have considered.

\section{ACKNOWLEDGMENTS}

We thank the referees for their valuable comments on the first version of our manuscript. This work was performed using HPC resources from CALMIP (Toulouse) under Allocation No. 2016-0510 and from GENCI-TGCC (Grant No. 201608s015).

${ }^{1}$ P. G. Szalay, T. Müller, G. Gidofalvi, H. Lischka, and R. Shepard, Chem. Rev. 112, 108 (2012).

${ }^{2}$ C. Hättig, W. Klopper, A. Köhn, and D. P. Tew, Chem. Rev. 112, 4 (2012).

${ }^{3}$ H. Lischka, R. Shepard, F. B. Brown, and I. Shavitt, Int. J. Quantum Chem. 20, 91 (2009).

${ }^{4}$ H. Lischka, R. Shepard, R. M. Pitzer, I. Shavitt, M. Dallos, T. Müller, P. G. Szalay, M. Seth, G. S. Kedziora, S. Yabushita, and Z. Zhang, Phys. Chem. Chem. Phys. 3, 664 (2001).

${ }^{5}$ J. Paldus and X. Li, Adv. Chem. Phys. 110, 1 (1999).

${ }^{6}$ L. Kong, K. Shamasundar, O. Demel, and M. Nooijen, J. Chem. Phys. 130, 114101 (2009).

${ }^{7}$ B. Jeziorski, Mol. Phys. 108, 3043 (2010).

${ }^{8}$ D. I. Lyakh, M. Musiał, V. F. Lotrich, and R. J. Bartlett, Chem. Rev. 112, 182 (2012).

${ }^{9}$ S. Epstein, Phys. Rev. 28, 695 (1926).

${ }^{10}$ R. K. Nesbet, Proc. R. Soc. A 230, 312 (1955).

${ }^{11}$ C. Angeli, R. Cimiraglia, S. Evangelisti, T. Leininger, and J.-P. Malrieu, J. Chem. Phys. 114, 10252 (2001).

${ }^{12}$ C. Angeli, R. Cimiraglia, and J.-P. Malrieu, J. Chem. Phys. 117, 9138 (2002).

${ }^{13}$ R. F. Fink, Chem. Phys. Lett. 428, 461 (2006).

${ }^{14}$ R. F. Fink, Chem. Phys. 356, 39 (2009).

${ }^{15}$ K. Andersson, P. A. Malmqvist, B. O. Roos, A. J. Sadlej, and K. Wolinski, J. Phys. Chem. 94, 5483 (1990).

${ }^{16}$ K. Andersson, P.-A. Malmqvist, and B. O. Roos, J. Chem. Phys. 96, 1218 (1992).

${ }^{17}$ P. Siegbahn, A. Heiberg, B. Roos, and B. Lévy, Phys. Scr. 21, 323 (1980).

${ }_{18}^{18}$ B. O. Roos, P. R. Taylor, and P. E. M. Siegbahn, Chem. Phys. 48, 157 (1980).

${ }^{19}$ P. E. M. Siegbahn, J. Almlöf, A. Heiberg, and B. O. Roos, J. Chem. Phys. 74, 2384 (1981).

${ }^{20}$ B. Huron, P. Rancurel, and J. P. Malrieu, J. Chem. Phys. 58, 5745 (1973).

${ }^{21}$ M. Caffarel, T. Applencourt, E. Giner, and A. Scemama, "Using CIPSI nodes in diffusion Monte Carlo," in Recent Progress in Quantum Monte Carlo (American Chemical Society (ACS), 2016), Chap. 2, pp. 15-46.

${ }^{22}$ G. H. Booth, A. J. W. Thom, and A. Alavi, J. Chem. Phys. 131, 054106 (2009).

${ }^{23}$ D. Cleland, G. H. Booth, C. Overy, and A. Alavi, J. Chem. Theory Comput. 8, 4138 (2012).

${ }^{24}$ F. R. Petruzielo, A. A. Holmes, H. J. Changlani, M. P. Nightingale, and C. J. Umrigar, Phys. Rev. Lett. 109, 230201 (2012).

${ }^{25}$ S. R. White, Phys. Rev. Lett. 69, 2863 (1992).

${ }^{26}$ S. R. White, Phys. Rev. B 48, 10345 (1993). 
${ }^{27}$ S. Sharma and G.-L. Chan, J. Chem. Phys. 136, 124121 (2012).

${ }^{28}$ E. Giner, C. Angeli, Y. Garniron, A. Scemama, and J.-P. Malrieu, J. Chem. Phys. 146, 224108 (2017).

${ }^{29}$ S. Evangelisti, J. P. Daudey, and J. P. Malrieu, Chem. Phys. 75, 91 (1983).

${ }^{30}$ S. Y. Willow, K. S. Kim, and S. Hirata, J. Chem. Phys. 137, 204122 (2012).

${ }^{31}$ S. Y. Willow, J. Zhang, E. F. Valeev, and S. Hirata, J. Chem. Phys. 140, 031101 (2014).

${ }^{32}$ S. Sharma, A. A. Holmes, G. Jeanmairet, A. Alavi, and C. J. Umrigar, J. Chem. Theory Comput. 13, 1595 (2017), pMID: 28263594.

${ }^{33}$ G. Jeanmairet, S. Sharma, and A. Alavi, J. Chem. Phys. 146, 044107 (2017).
${ }^{34}$ A. Szabo and N. S. Ostlund, Modern Quantum Chemistry (McGraw-Hill, New York, 1989).

${ }^{35}$ A. Scemama and E. Giner, e-print arXiv:1311.6244 [physics.comp-ph] (2013).

${ }^{36}$ T. H. Dunning, J. Chem. Phys. 90, 1007 (1989).

${ }^{37}$ P. Hudak, ACM Comput. Surv. 21(3), 359 (1989).

${ }^{38}$ A. Scemama, T. Applencourt, Y. Garniron, E. Giner, and M. Caffarel (2017). "Quantum Package," Zenodo V. 1.1, Dataset http://dx.doi.org/ 10.5281/zenodo.825876.

${ }^{39}$ N. B. Balabanov and K. A. Peterson, J. Chem. Phys. 123, 064107 (2005).

${ }^{40}$ M. W. Schmidt, K. K. Baldridge, J. A. Boatz, S. T. Elbert, M. S. Gordon, J. H. Jensen, S. Koseki, N. Matsunaga, K. A. Nguyen, S. Su, T. L. Windus, M. Dupuis, and J. A. Montgomery, J. Comput. Chem. 14, 1347 (1993). 